\title{
Risk Factors for Early Postoperative Small Bowel Obstruction after Anterior Resection for Rectal Cancer: Methodological Issues
}

\author{
Jie Weng ${ }^{1} \cdot \mathrm{He} \mathrm{Wu}^{1} \cdot$ Zhiyi Wang $^{1,2}$
}

Published online: 13 November 2017

(C) Société Internationale de Chirurgie 2017

\section{Dear Editor,}

We were interested to read the paper authored by Suwa and colleagues published in the World Journal of Surgery in 2017 [1]. Their study was to assess the risk factors for small bowel obstruction (SBO) in early postoperative period after anterior resection (AR) for rectal cancer. The results provide evidence that the $\mathrm{D} 3$ lymph node dissection and DI formation are independent risk factors for EPSBO in AR [1]; however, some methodological issues should be noticed.

In this study, multivariate analysis was performed using the factors that were considered significant in the univariate analysis (factors with $p$ values less than 0.05 ). The risk factors with $p$ values less than 0.05 which have large effect on outcome and $p$ values less than 0.2 may have a relatively small effect on outcome. If we selected only those with $p$ value $<0.05$ in multivariable analysis, the effect of such predictors will be overestimated and in following the prediction power will be decreased. So, independent variables with $p$ values less than 0.2 should be selected to be imported into the multivariable model. Otherwise, it can induce testimation bias in the results [2].

In the study, some odds ratios (ORs) and CIs were relatively large such as $17.25(3.49-312.55)$ for low anterior resection and 13.61 (2.75-246.69) for D3 lymph node dissection in Table 2 . This is probably due to the

Zhiyi Wang

wzy1063@126.com

1 Department of Emergency Medicine, The Second Affiliated Hospital and Yuying Children's Hospital of Wenzhou Medical University, Wenzhou 325000, China

2 Department of General Practice, The Second Affiliated Hospital and Yuying Children's Hospital of Wenzhou Medical University, Wenzhou 325000, China multicollinearity [2]. Although, the authors have mentioned it in their study, the multivariate model may be affected by bias because of the multicollinearity [2].

The authors concluded that D3 lymph node dissection and DI formation are independent risk factors for early postoperative $\mathrm{SBO}$ in AR. However, the prediction models were not be validated through using some appropriate methods such as cross-validation and bootstrapping, otherwise, these results would be optimistic interpretation. Correcting optimism problem in study with small sample size have been more emphasized [3].

Compliance with ethical standards

Conflict of interest The authors declare that they have no conflict of interest.

\section{References}

1. Suwa K, Ushigome T, Ohtsu M et al (2017) Risk factors for early postoperative small bowel obstruction after anterior resection for rectal cancer. World J Surg. https://doi.org/10.1007/s00268-0174152-y

2. Steyerberg E (2008) Clinical prediction models: a practical approach to development, validation, and updating. Springer, Berlin

3. Smith GC, Seaman SR, Wood AM et al (2008) Correcting for optimistic prediction in small data sets. Am J Epidemiol 180:318-324 